\title{
CORRELATION ANALYSIS OF GSTP1 GENE POLYMORPHISM WITH MORBIDITY OF PRIMARY METASTATIC COLORECTAL CANCER
}

\author{
Yuri Bazhora \\ Department of clinical immunology, genetics and medical biology ${ }^{1}$ \\ Sergii Chetverikov \\ Surgery department No.4 with oncology course ${ }^{2}$ \\ chetverikov@rambler.ru \\ Viacheslav Onyshchenko \\ Department of simulation medicine ${ }^{1}$ \\ vicedeanodessa@gmail.com \\ Mykhailo Chetverikov \\ Internship doctor ${ }^{2}$ \\ chetvericov@yahoo.com \\ Valeriia Chetverikova-Ovchynnyk \\ Internship doctor ${ }^{2}$ \\ ovchinnik.val@yahoo.com \\ ${ }^{1}$ Odessa National Medical University \\ 2 Valichovskiy lane, Odessa, Ukraine, 65082 \\ ${ }^{2}$ Center of Reconstructive and Restorative Medicine (University Clinic) of \\ Odessa National Medical University \\ 8 Tenista str., Odessa, Ukraine, 65009
}

\begin{abstract}
Colorectal cancer (CRC) is one of the most common malignancies. Susceptibility to malignant processes is mediated by genetically driven differences in the effectiveness of detoxification of potential carcinogens. One of the factors that may influence the risk of CRC is the glutathione-S-transferase (GST) gene family that encodes glutathione transferase enzymes. The GSTP1 gene is expressed both in normal and pathological conditions. Determining its specific alleles may be a marker of CRC.

The aim of the research - to study GSTP1 gene polymorphism, which is likely to be more common among patients with primary metastatic colorectal cancer compared with healthy population.

Materials and methods. The study involved 12 patients with primary metastatic colorectal cancer aged 43 to 72 years, the control group was represented by a sample of 31 people without cancer.

Results. The incidence of advanced CRC in the presence of GSPT1 Val/Val (aa) polymorphism is statistically significantly higher than in the presence of Ile/Ile (AA) and Ile/Val (Aa) GSPT1 polymorphism.

Conclusions. Among people with GSTP1 Val/Val (aa) polymorphism, primary CRC is 4.4 times more likely than among people with GSPT1 Ile/Ile (AA) and Ile/Val (Aa) polymorphisms, which are statistically significant $(\mathrm{p}<0.05)$. The obtained results indicate the possibility of conducting a genetic study of GSTP1 polymorphism to form groups of potential risk of CRC.
\end{abstract}

Keywords: glutathione-S-transferase P1, metastatic colorectal cancer, genetic polymorphism.

DOI: $10.21303 / 2504-5679.2020 .001196$

\section{Introduction}

Colon cancer is one of the most common malignancies and ranks third in the list of the most commonly diagnosed malignancies among men and second among women. Approximately 1.8 million first-time cases of colorectal cancer (CRC) are reported annually. According to the WHO GLOBOCAN database, in 2018, almost 881 thousand people died from CRC [1]. Among them, $24 \%$ of patients are diagnosed with primary advanced CRC, and another $48 \%$ of patients 
will have metastases, which contributes to high mortality rates. According to ESMO, the 5-year survival rate in patients with CRC is approaching $60 \%$ [2]. According to the National Cancer Registry of Ukraine for 2017, the incidence rate of patients with colorectal cancer in 2017 compared to 2016 is $5 \%$ [3]. CRC mortality rates have been declining gradually since the mid-1980s in the United States and many other developed countries [4, 5]. This improvement, at least in part, may be related to the detection and removal of colon polyps, early-stage CRC detection, more effective primary and adjuvant treatments, and the improvement of instrumental and laboratory diagnostic methods, in particular molecular studies. Among them, those predominate that relate to the genetic conditioning of cancer and the pathways of carcinogenesis. The accumulated evidence suggests that susceptibility to malignant processes, including CRC, is mediated by genetically driven differences in the effectiveness of detoxification of potential carcinogens. The study of the impact of specific genes on the risk of CRC development is a relevant area of research. One such factor is the family of glutathione-S-transferase (GST) genes encoding glutathione transferase enzymes. GST are phase II detoxification enzymes that catalyze the conjugation of electrophilic compounds with reduced glutathione, playing a central role in the cellular detoxification of various groups of exogenous and endogenous harmful compounds. Based on their biochemical properties, human GSTs are divided into $\alpha, \kappa, \mu, \omega, \pi, \theta, \zeta$ and microsomal classes. GST $\pi$ (hereinafter - GSTP1) is often overexpressed in many malignant processes, including tumors of the brain, breast [6], ovaries, esophagus, stomach [7], pancreas, colon, skin, kidneys, lungs [8], bile ducts, lymphatic and hematopoietic systems. GST gene polymorphism can influence the risk of CRC by modifying the effects of carcinogenic food-related agents on the intestinal mucosa. The GSTP1 gene is expressed as normal and pathological, however, the determination of its specific alleles can be a marker of CRC. According to existing studies, an increase in the level of the GSTP1 enzyme in tumors of the colon may indicate high resistance to anticancer drugs $[9,10]$.

The aim of the research - to study GSTP1 gene polymorphism, which is likely to be more common among patients with primary metastatic colorectal cancer compared with healthy population.

\section{Materials and methods of the research}

The study involved 12 patients with newly diagnosed metastatic colorectal cancer aged 43 to 72 years (mean age 65.1 years) who underwent special treatment at the University Clinic of Odessa National Medical University from January to August 2019. Diagnosis in all patients was confirmed histologically and immunohistochemically; the spread of the process was determined intraoperatively and by methods of preoperative intrascopic examinations. In assessing GSTP1 gene polymorphism, the control group was represented by a sample of 31 people without oncopathology (mean age 62.3 years) living in Odessa. The nuclear material of patients' leukocytes of venous blood was studied. Samples were presented by patients of Ukrainian nationality.

The GSTP1 gene is characterized by a number of polymorphisms, the most common is the 105Ile/Val polymorphism in the fifth exon and 114Ala / Val in the sixth exon [11]. The 105Ile/Val polymorphism is associated with the single-nucleotide substitution of $313 \mathrm{~A}>\mathrm{G}$ and relates to a hydrophobic substrate binding site that has a significant effect on the course of biochemical reactions. The Val variant three times reduces the detoxification activity of the enzyme against xenobiotics. EX5-1 5'-GTAGTTTGCCCAAGGTCAAG-3' primers starting from 2306 nucleotide pairs of the complete GSTP1 sequence and EX5-2 5'-AGCCACCTGAGGGGTAAG-3' primers starting at 2721 nuclei were used to amplify the polymorphic version of exon 5. $8 \mu$ of isolated DNA was added to a polymerase chain reaction (PCR) mixture containing $0.5 \mathrm{U}$ Taq-polymerase, $11 \mathrm{pmol}$ of each primer, $1.5 \mu \mathrm{l}$ of 10 'PCR buffer $\left(25 \mathrm{~mm} \mathrm{MgCl}_{2}\right)$ and $2.5 \mu \mathrm{l}$ of $10 \mathrm{hdNTPmix}$ in a final volume of $15 \mu$ l. "Hot Start" was used to prevent nonspecific priming in the first PCR cycle. After initial denaturation for $12 \mathrm{~min}$ at $95^{\circ} \mathrm{C}$, the following 15 cycles of PCR were performed (denaturation for $30 \mathrm{~s}$ at $95^{\circ} \mathrm{C}$, annealing for $30 \mathrm{~s}$ at $58^{\circ} \mathrm{C}$, elongation for $60 \mathrm{~s}$ at $72{ }^{\circ} \mathrm{C}$ ), followed by 25 cycles amplification (denaturation for $30 \mathrm{~s}$ at $95^{\circ} \mathrm{C}$, annealing for $30 \mathrm{~s}$ at $55^{\circ} \mathrm{C}$, elongation for $60 \mathrm{~s}$ at $72{ }^{\circ} \mathrm{C}$ ) and one elongation cycle at $72{ }^{\circ} \mathrm{C}$ for five minutes. PCR products were cut for two hours at $37{ }^{\circ} \mathrm{C}$ with five units of Alw26I (Fermentas Inc, Vilnius, Lithuania). During $2 \%$ agarose gel electrophoresis 
in the presence of the 105Ile allele in the homozygous state, fragments of size 329 and 104 pairs of nucleotides are observed, in the presence of the allele 105Val in the homozygous state, fragments in the size of 222 and 107 nucleotide pairs, in heterozygotes - all four DNA fragments (Fig. 1).

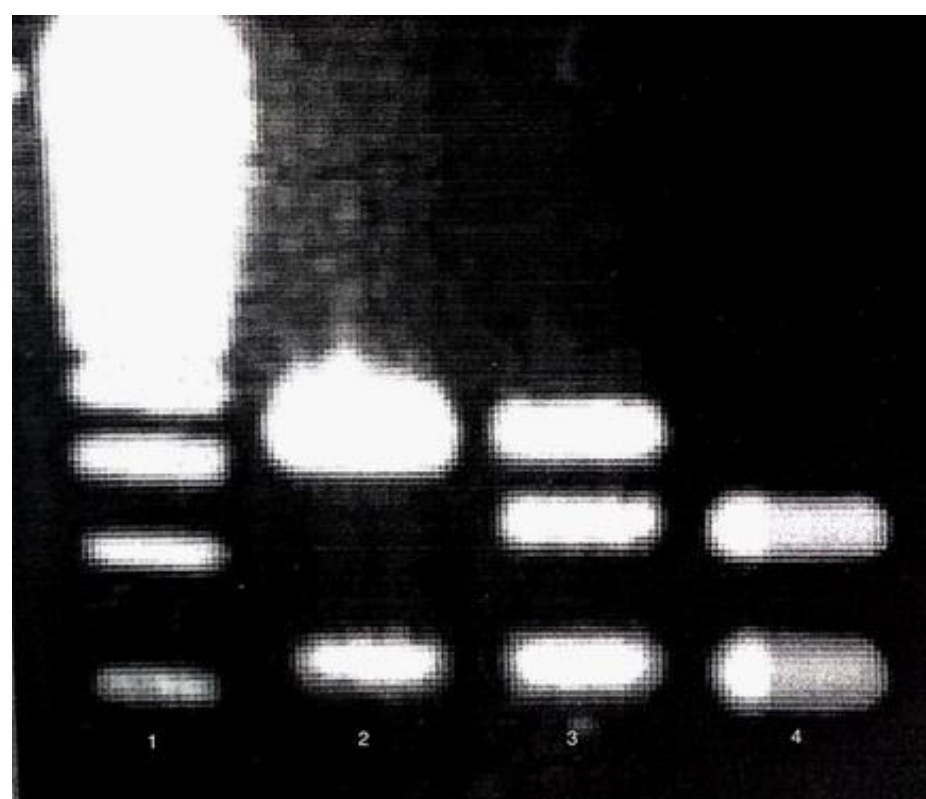

Fig. 1. Electrophoregram of human DNA samples containing the 105Ile/Val polymorphism of the GSTP1 gene: 1 - molecular steps; 2 - homozygote 105Ile/Ile; 3 - heterozygote 105Ile/Val; 4 - homozygote 105Val/Val.

The study was conducted within the framework of joint scientific work of the Department of Surgery No. 4 with the oncology course and the Department of Medical Biology of the Odessa National Medical University. Compliance with the World Medical Association Code of Ethics WMA Declaration of Helsinki - Ethical Principles for Medical Research Involving Human Subjects, 2013 (minutes of the meeting of the Committee on Bioethics of the Odessa National Medical University No. 123A dated 29.08.2019) was determined. All study participants were informed and agreed to the processing of their clinical data and to participate in the research process.

The statistical analysis of the data obtained was performed using GNU PSPP version 1.2.0 using Fisher's two-sided exact $\mathrm{P}$ test.

\section{Results}

The incidence of GSTP1 gene polymorphism among CRC patients and controls was presented in Table 1.

Table 1

The incidence of GSTP1 gene polymorphism among CRC patients and control group

\begin{tabular}{cccc}
\hline Genotypes & Ile/Ile (AA) & Ile/Val (Aa) & Val/Val (aa) \\
\hline $\begin{array}{c}\text { Patients } \\
\mathrm{n}=12\end{array}$ & $4(33.33 \%)$ & $2(16.67 \%)$ & $6(50.00 \%)$ \\
$\begin{array}{c}\text { Control } \\
\mathrm{n}=31\end{array}$ & $17(54.84 \%)$ & $12(38.71 \%)$ & $2(6.45 \%)$
\end{tabular}

According to Table 1, in the sample of patients with initially diagnosed metastatic CRC homozygotes for the Val allele were in $6(50.00 \%)$ studied, while in the control group in $2(6.45 \%)$ persons. 
Tables 2-4 present a comparison of the frequency of occurrence of GSTP1 Ile/Ile (AA) polymorphism with Ile/Val (AA)+Val/Val (AA), Ile/Val (AA) with Ile/Ile (AA)+Val/Val (aa) and $\mathrm{Val} / \mathrm{Val}$ (aa) with Ile/Ile (AA)+Ile/Val (AA) respectively in patients with initially diagnosed metastatic CRC and in the control group, statistical significance using Fisher's two-sided exact P test, and also the significance of the RR relationship (95\% CI).

Table 2

Comparison of the incidence of GSTP1 Ile/Ile (AA) polymorphism with Ile/Val (AA)+Val/Val (AA) among CRC patients and control group

\begin{tabular}{|c|c|c|}
\hline Genotypes & Ile/Ile (AA) & Ile/Val (Aa)+Val/Val (aa) \\
\hline $\begin{array}{c}\text { Patients } \\
n=12\end{array}$ & $4(33.33 \%)$ & $8(66.67 \%)$ \\
\hline $\begin{array}{c}\text { Control } \\
n=31\end{array}$ & $17(54.84 \%)$ & $14(45.16 \%)$ \\
\hline Fisher's P-test & \multicolumn{2}{|c|}{$0,31015(\mathrm{p}=0.05)$} \\
\hline $\mathrm{RR}(95 \% \mathrm{CI})$ & \multicolumn{2}{|c|}{$0.524(0.185-1.483)$} \\
\hline
\end{tabular}

According to the results in Table 2, the Fisher P-test, which was determined when calculating the incidence of Ile/Ile (AA) polymorphism compared with Ile/Val (Aa) and Val/Val (aa) in patients with metastatic CRC and in the control group was 0.31015 , thus Fisher's P-test $>0.05$. The incidence of advanced CRC in the presence of GSPT1 Ile/Ile (AA) polymorphism is lower than in the presence of GSPT1 Ile/Val (AA)+Val/Val (AA) polymorphism, but the difference is not statistically significant. The relative risk indicator is 0.524 at $95 \% \mathrm{CI}(0.185-1.483)$. The significance level of this relationship is greater than 0.05 , since $95 \% \mathrm{CI}$ includes one, so reducing the likelihood of primary metastatic CRC with GSPT1 Ile/Ile (AA) is not statistically significant.

According to the results given in Table 3, which were determined when calculating the incidence of polymorphism Ile/Val (AA) compared with Ile/Ile (AA)+Val/Val (AA) in patients with $\mathrm{CRC}$ and in the control group was 0.27852 , thus Fisher's P-test $>0.05$. The incidence of metastatic CRC in the presence of the Ile/Val (AA) polymorphism is lower than in the presence of the GSPT1 Ile/Val (AA)+Val/Val (AA) polymorphism, but the difference is not statistically significant. The relative risk indicator is 0.414 at $95 \% \mathrm{CI}(0.104-1.643)$. The significance level of this relationship is greater than 0.05 , since $95 \%$ CI includes one, so reducing the likelihood of primary diagnosed metastatic CRC with GSPT1 Ile/Val (AA) is not statistically significant.

Table 3

Comparison of the incidence of GSTP1 Ile/Val (AA) polymorphism with Ile/Ile (AA)+Val/Val (AA) among CRC patients and control group

\begin{tabular}{|c|c|c|}
\hline Genotypes & Ile/Val (Aa) & Ile/Ile (AA)+Val/Val (aa) \\
\hline $\begin{array}{c}\text { Patients } \\
n=12\end{array}$ & $2(16.67 \%)$ & $10(83.33 \%)$ \\
\hline $\begin{array}{c}\text { Control } \\
n=31\end{array}$ & $12(38.71 \%)$ & $19(61.29 \%)$ \\
\hline Fisher's P-test & \multicolumn{2}{|c|}{$0.27852(\mathrm{p}=0,05)$} \\
\hline $\mathrm{RR}(95 \% \mathrm{CI})$ & \multicolumn{2}{|c|}{$0.414(0.104-1.643)$} \\
\hline
\end{tabular}

According to the results in Table 4 Fisher's P-test, which was determined when calculating the frequency of occurrence of Ile/Ile (AA) and Ile/Val (Aa) polymorphism in comparison with Val/Val (aa) in patients with CRC and in the control group was 0.00314 , that is, the value of the Fisher P-test $<0.05$. The incidence of advanced CRC in the presence of GSPT1 Val/Val (aa) polymorphism is statistically significantly higher than in the case of GSPT1 Ile/Ile (AA) and Ile/Val (Aa) polymorphism. 
Table 4

Comparison of incidence of GSTP1 Val/Val (aa) polymorphism with Ile/Ile (AA)+Ile/Val (AA) among CRC patients and controls

\begin{tabular}{ccc}
\hline Genotypes & Val/Val (aa) & Ile/Ile (AA)+Ile/Val (Aa) \\
\hline $\begin{array}{c}\text { Patients } \\
\mathrm{n}=12\end{array}$ & $6(50.00 \%)$ & $6(50.00 \%)$ \\
Control & $2(6.45 \%)$ & $29(93.55 \%)$ \\
$\mathrm{n}=31$ & & $0.00314(\mathrm{p}=0,05)$ \\
Fisher's P-test & & $4.375(1.906-10.043)$ \\
RR $(95 \% \mathrm{CI})$ & &
\end{tabular}

According to the calculations in Table 4, the relative risk indicator for the presence of GSTP1 Val/Val (aa) polymorphism is 4.375 at $95 \%$ CI (1.906-10.043). This indicates that there is a direct relationship between the presence of GSTP1 Val/Val (aa) polymorphism and the likelihood of developing metastatic CRC. Among people with GSTP1 Val/Val (aa) polymorphism, primary-spread CRC is 4.375 times more likely than among people with GSPT1 Ile/Ile (AA) and Ile/Val (Aa) polymorphism. The significance level of this relationship corresponds to $\mathrm{p}<0.05$, since $95 \%$ CI does not include one.

\section{Disscussion}

Epidemiological studies indicate that individuals with the expression of different alleles of the GSTP1 gene are susceptible to chemical carcinogens, because polymorphism of the GSTP1 gene can cause changes in the expression or structure of the enzyme, leading to a decrease in the efficiency of the catalytic function of variants of the enzyme, ie, insufficient detoxification of carcinogens and, therefore, affect the development of CRC. GSTP1 is expressed normally in epithelial tissue, but there are works on its overexpression in colon cancer [12]. Functional polymorphism of the GSTP1 gene, which reduces enzymatic activity, involves the replacement of A-G in 5 exons and the conversion of isoleucine to valine at position 105 of the amino acid chain (Ile105Val) [13]. In Rodrigues-Fleming GH et al., 2018, GST genes were studied in patients with sporadic colorectal cancer, identifying a direct relationship between the presence of the wild-type GSTP1 Ile105Val polymorphism and the smoking risk of CRC [14]. Carriers of these mutations have a reduced detoxification function of the enzyme in relation to carcinogens, so there is an increased risk of a malignant process $[11,15]$.

In the study, we identified a possible correlation of common GSTP1 polymorphism with the presence of primary advanced CRC. The distribution of the GSPT1 genotype among patients was Ile/Ile (AA) $33.33 \%$, Ile/Val (AA) $16.67 \%$ and $\mathrm{Val} / \mathrm{Val}$ (AA) $50.0 \%$, whereas in the control group Ile/Ile (AA) $54.84 \%$, Ile/Val (AA) $38.71 \%$ and Val/Val (AA) $6.45 \%$ (Table 1). In contrast to the described results, in the work of Gorukmez O. et al., 2016, the GSTP1 Ile/Ile (AA) gene polymorphism was more common in CRC patients, and the Ile/Val (AA) and Val/Val (AA) genotypes were more common in the control group [12]. When comparing the incidence of GSPT1 Val/Val (aa) polymorphism with Ile/Ile (AA)+Ile/Val (AA) polymorphisms, the obtained results indicate that there is a direct relationship between GSPT1 Val/Val (aa) polymorphism and the probability of primary diagnosed metastatic CRC (Table 4). The incidence of advanced CRC in the presence of GSPT1 $\mathrm{Val} / \mathrm{Val}$ (aa) polymorphism is statistically significantly higher than in the presence of Ile/Ile (AA) and Ile/Val (Aa) GSPT1 polymorphism. Among people with GSTP1 Val/Val (aa) polymorphism, primary diagnosed CRC is 4.375 times more likely than among people with GSPT1 Ile/Ile (AA) and Ile/Val (Aa) polymorphisms, which are statistically significant $(\mathrm{p}<0.05)$ (Table 4). Similar results were obtained in the study by Khabaz M. N. et al., 2016, the incidence of the GSPT1 Val/Val (aa) genotype in colorectal cancer cases was three times higher than in the control groups, but the results were not statistically significant [16]. In contrast, in the study of GSTP1 polymorphism and the risk of colorectal cancer among the Polish population, the genotype frequency of Ile/Ile (AA), Ile/Val (Aa) and Val/Val (AA) was respectively $42.1 \%, 48.2 \%, 9.6 \%$ in patients and $48.1 \%$, 
$42.3 \%$ and $9.6 \%$ in control groups [17]. In another study of GSTP1 gene polymorphism in an ethnic Kashmiri population, a homozygous variant of the Val/Val (aa) genotype was associated with an increased risk of CRC [18]. In the study of GSTP1 polymorphism in the Tunisian population, a direct correlation of the homozygous variant of the Val/Val (aa) genotype with the incidence of CRC was also identified [19].

In contrast, the results of the Cong $\mathrm{N}$ et. al, 2014 in the Chinese population showed no association between GSTP1 Val/Val (aa) polymorphism and CRC risk [20].

Study limitations. The main limitation of the study is the identification of GSTP1 gene polymorphism in this sample, not taking into account the possible presence of other genetic mutations associated with the risk of CRC. In addition, despite the statistically reliable data obtained, an increase in the sample size and the distribution of patients into different subgroups by gender and age would allow to obtain data that are more accurate.

Prospects for futher research. In the future, it is planned to analyze the dependence of GSTP1 gene polymorphism and the results of special treatment (combination treatment in the form of cytoreductive surgery and subsequent adjuvant PCT and exclusively PCT) in patients with initially diagnosed CRC, based on an assessment of the duration of their disease-free period and overall survival. It is also planned to study the correlation of the degree of differentiation of CRC with polymorphisms of the GSTP1 gene.

\section{Conclusions}

In this study, genetic factors that can play a significant role in the pathogenesis of CRC are analyzed. According to the analyzed data, among people with GSTP1 Val/Val (aa) polymorphism, the primary diagnosed metastatic CRC is statistically significantly more frequent than among people with GSPT1 Ile/Ile (AA) and Ile/Val (Aa) polymorphisms. The obtained results indicate the possibility of conducting a genetic study of GSTP1 polymorphism to form groups of potential risk of CRC. Understanding the role of GST gene polymorphism and their interaction with other factors in the process of carcinogenesis can improve the tactics of diagnosis and prevention of various types of malignant processes. Thus, our findings provide the basis for future epidemiological and clinical studies.

\section{Conflict of interests}

The authors declare that they have no conflicts of interest.

\section{References}

[1] Ferlay, J., Soerjomataram, I., Ervik, M., Dikshit, R., Eser, S., Mathers, C. et al. (2018). GLOBOCAN 2018 v1.0, Cancer Incidence and Mortality Worldwide: IARC CancerBase No. 11. Lyon: International Agency for Research on Cancer.

[2] Van Cutsem, E., Cervantes, A., Nordlinger, B., Arnold, D. (2014). Metastatic colorectal cancer: ESMO Clinical Practice Guidelines for diagnosis, treatment and follow-up. Annals of Oncology, 25, 1-9. doi: http://doi.org/10.1093/annonc/mdu260

[3] Fedorenko, Z. P., Goulak, L. O., Gorokh, Y. L., Ryzhov, A. Y., Soumkina, E. V., Koutsenko, L. B. (2018). Cancer in Ukraine. Available at: www.ncru.inf.ua/publications/ Last accessed: 21.08.2017

[4] Siegel, R. L., Miller, K. D., Jemal, A. (2019). Cancer statistics, 2019. CA: A Cancer Journal for Clinicians, 69 (1), 7-34. doi: http://doi.org/10.3322/caac. 21551

[5] Cronin, K. A., Lake, A. J., Scott, S., Sherman, R. L., Noone, A.-M., Howlader, N. et. al. (2018). Annual Report to the Nation on the Status of Cancer, part I: National cancer statistics. Cancer, 124 (13), 2785-2800. doi: http://doi.org/10.1002/cncr.31551

[6] Louie, S. M., Grossman, E. A., Crawford, L. A., Ding, L., Camarda, R., Huffman, T. R. et. al. (2016). GSTP1 Is a Driver of Triple-Negative Breast Cancer Cell Metabolism and Pathogenicity. Cell Chemical Biology, 23 (5), 567-578. doi: http:// doi.org/10.1016/j.chembiol.2016.03.017

[7] Chen, Z. H., Xian, J. F., Luo, L. P. (2017). Association between GSTM1, GSTT1, and GSTP1 polymorphisms and gastric cancer risk, and their interactions with environmental factors. Genetics and Molecular Research, 16 (1). doi: http://doi.org/10.4238/ gmr16018877

[8] Shahsavari, G., Amiri, A., Shamaei, M., Adibhesami, G., Emami Razavi, A., Birjandi, M., Pourabdollah, M. (2019). The Relation between Polymorphisms in Exon 5 and Exon 6 of GSTP1 Gene and the Risk of Lung Cancer in Iranian People. Asian Pacific Journal of Cancer Prevention, 20 (5), 1503-1509. doi: http://doi.org/10.31557/apjcp.2019.20.5.1503 
[9] Sun, Y., Pan, J., Tong, X., Chen, E., Yan, W., Wu, M. et. al. (2019). Glutathione S-transferases genes variants and chemotherapy efficacy in gastrointestinal cancer patients: a meta-analysis based on 50 pharmacogenetic studies. Journal of Cancer, 10 (13), 2915-2926. doi: http://doi.org/10.7150/jca.31130

[10] Shen, X., Wang, J., Yan, X., Ren, X., Wang, F., Chen, X., Xu, Y. (2016). Predictive value of GSTP1 Ile105Val polymorphism in clinical outcomes of chemotherapy in gastric and colorectal cancers: a systematic review and meta-analysis. Cancer Chemotherapy and Pharmacology, 77 (6), 1285-1302. doi: http://doi.org/10.1007/s00280-016-3047-1

[11] Sharma, A., Pandey, A., Sharma, S., Chatterjee, I., Mehrotra, R., Sehgal, A., Sharma, J. K. (2014). Genetic polymorphism of glutathione S-transferase P1 (GSTP1) in Delhi population and comparison with other global populations. Meta Gene, 2, 134-142. doi: http://doi.org/10.1016/j.mgene.2013.12.003

[12] Zhang, R., Kang, K. A., Piao, M. J., Kim, K. C., Zheng, J., Yao, C. W. et. al. (2014). Epigenetic alterations are involved in the overexpression of glutathione S-transferase $\pi-1$ in human colorectal cancers. International Journal of Oncology, 45 (3), 1275-1283. doi: http://doi.org/10.3892/ijo.2014.2522

[13] Gorukmez, O., Yakut, T., Gorukmez, O., Sag, S. O., Topak, A., Sahinturk, S., Kanat, O. (2016). Glutathione S-transferase T1, M1 and P1 Genetic Polymorphisms and Susceptibility to Colorectal Cancer in Turkey. Asian Pacific Journal of Cancer Prevention, 17 (8), 3855-3859.

[14] Rodrigues-Fleming, G. H., Fernandes, G. M. de M., Russo, A., Biselli-Chicote, P. M., Netinho, J. G., Pavarino, É. C., GoloniBertollo, E. M. (2018). Molecular evaluation of glutathione S transferase family genes in patients with sporadic colorectal cancer. World Journal of Gastroenterology, 24 (39), 4462-4471. doi: http://doi.org/10.3748/wjg.v24.i39.4462

[15] Saeed, H. M., Alanazi, M. S., Nounou, H. A., Shalaby, M. A., Semlali, A., Azzam, N. et. al. (2013). Cytochrome P450 1A1, 2E1 and GSTM1 Gene Polymorphisms and Susceptibility to Colorectal Cancer in the Saudi Population. Asian Pacific Journal of Cancer Prevention, 14 (6), 3761-3768. doi: http://doi.org/10.7314/apjcp.2013.14.6.3761

[16] Khabaz, M. N., Al-Maghra bi, J. A., Nedjadi, T., Gar, M. A., Bakarman, M., Gazzaz, Z. J., Ibrahim, A. M. (2016). Does Val/Val genotype of GSTP1 enzyme affects susceptibility to colorectal cancer in Saudi Arabia? Neuro Endocrinol Lett, 37 , 46-52.

[17] Klusek, J., Nasierowska-Guttmejer, A., Kowalik, A., Wawrzycka, I., Lewitowicz, P., Chrapek, M., Głuszek, S. (2018). GSTM1, GSTT1, and GSTP1 polymorphisms and colorectal cancer risk in Polish nonsmokers. Oncotarget, 9 (30), 21224-21230. doi: http://doi.org/10.18632/oncotarget.25031

[18] Rashid, F., Nissar, S., Sameer, A., Rasool, R., Chowdri, N. (2019). Promoter methylation and Ile105val polymorphism of GSTP1 gene in the modulation of colorectal cancer risk in ethnic Kashmiri population. Indian Journal of Cancer, 56 (3), 248-253. doi: http://doi.org/10.4103/ijc.ijc_11_18

[19] Kassab, A., Msolly, A., Lakhdar, R., Gharbi, O., Miled, A. (2013). Polymorphisms of glutathione-S-transferases M1, T1, P1 and susceptibility to colorectal cancer in a sample of the Tunisian population. Medical Oncology, 31 (1). doi: http://doi.org/10.1007/ s12032-013-0760-z

[20] Cong, N., Liu, L., Xie, Y., Shao, W., Song, J. (2014). Association between Glutathione S-Transferase T1, M1, and P1 Genotypes and the Risk of Colorectal Cancer. Journal of Korean Medical Science, 29 (11), 1488-1492. doi: http://doi.org/10.3346/ jkms.2014.29.11.1488 